\title{
An Arabidopsis thaliana gene for methylsalicylate biosynthesis, identified by a biochemical genomics approach, has a role in defense
}

Feng Chen ${ }^{1}$, John C. D’Auria ${ }^{1, \dagger}$, Dorothea Tholl ${ }^{1,2}$, Jeannine R. Ross ${ }^{3}$, Jonathan Gershenzon ${ }^{2}$, Joseph P. Noel ${ }^{3}$ and Eran Pichersky ${ }^{1, *}$

${ }^{1}$ Department of Molecular, Cellular, and Developmental Biology, University of Michigan, Ann Arbor, MI 48109, USA,

${ }^{2}$ Max Planck Institute for Chemical Ecology, Beutenberg Campus, Winzerlaer Strasse 10, D-07745 Jena, Germany, and

${ }^{3}$ Structural Biology Laboratory, The Salk Institute for Biological Studies, La Jolla, CA 92037, USA

Received 26 June 2003; revised 8 August 2003; accepted 15 August 2003.

*For correspondence (fax +1 734647 0884; e-mail lelx@umich.edu).

†Present address: Max Planck Institute for Chemical Ecology, Beutenberg Campus, Winzerlaer Strasse 10, D-07745 Jena, Germany.

\begin{abstract}
Summary
Emission of methylsalicylate (MeSA), and occasionally of methylbenzoate (MeBA), from Arabidopsis thaliana leaves was detected following the application of some forms of both biotic and abiotic stresses to the plant. Maximal emission of MeSA was observed following alamethicin treatment of leaves. A gene (AtBSMT1) encoding a protein with both benzoic acid (BA) and salicylic acid (SA) carboxyl methyltransferase activities was identified using a biochemical genomics approach. Its ortholog (AIBSMT1) in A. lyrata, a close relative of $A$. thaliana, was also isolated. The AtBSMT1 protein utilizes SA more efficiently than BA, whereas AIBSMT1 catalyzes the methylation of SA less effectively than that of BA. The AtBSMT1 and AIBSMT1 genes showed expression in leaves under normal growth conditions and were more highly expressed in the flowers. In A. thaliana leaves, the expression of AtBSMT1 was induced by alamethicin, Plutella xylostella herbivory, uprooting, physical wounding, and methyl jasmonate. SA was not an effective inducer. Using a $\beta$-glucuronidase (GUS) reporter approach, the promoter activity of AtBSMT1 was localized to the sepals of flowers, and also to leaf trichomes and hydathodes. Upon thrip damage to leaves, AtBSMT1 promoter activity was induced specifically around the lesions.
\end{abstract}

Keywords: Arabidopsis lyrata, benzenoids, emission, gene family, methyltransferase, volatile esters.

Introduction

Many plants, particularly those pollinated by moths, emit floral scents that contain benzenoid esters such as methylsalicylate (MeSA) and methylbenzoate (MeBA) to attract these insect pollinators (Knudsen et al., 1993). The moth Hyles lineata showed strong electroantennogram response to MeSA, indicating that it can detect MeSA at very low concentrations (Raguso et al., 1996). Some flowers, such as Anthirrhinum majus (snapdragon) and Stephanotis floribunda, show a diurnal rhythm of MeSA and/or MeBA emission, with peak emission coinciding with the peak activity of the respective pollinator, and this rhythm appears to be controlled by a circadian clock (Kolosova et al., 2001; Pott et al., 2002).

Reports of the synthesis and/or emission of benzenoid esters from leaves are fewer, but this may simply reflect the paucity of studies of vegetative volatiles compared with ๑ 2003 Blackwell Publishing Ltd floral scent analyses. Shulaev et al. (1997) reported that tobacco leaves infected with the tobacco mosaic virus (TMV) synthesized and emitted MeSA from both infected and non-infected leaves, and that under very high concentrations of airborne MeSA, neighboring plants could absorb MeSA, convert it to salicylic acid (SA), and thereby induce SA-mediated defense response against TMV (Shulaev et al., 1997). More recently, leaves of Arabidopsis thaliana attacked by herbivorous insects were shown to emit MeSA as well as many other volatiles (Van Poecke et al., 2001). Although the specific biological function of MeSA in this case is currently unknown, it was hypothesized that the emission of a blend of volatiles is part of an ecological mechanism to attract predatory insects that prey on the herbivorous insects causing the initial damage to the plant (Van Poecke et al., 2001). MeSA and MeBA also function as 
animal and insect toxins that exert their deleterious effects internally after being ingested by the organism (Lindberg et al., 2000). Moreover, MeSA has also been shown to possess microbicidal properties (Demirci et al., 2000).

MeSA and MeBA are synthesized in planta via a reaction catalyzed by methyltransferases whereby a methyl group is transferred from the donor molecule $S$-adenosine-Lmethionine (SAM) to the carboxyl group of SA or benzoic acid (BA), respectively. SA methyltransferase (SAMT) activity has been characterized in flowers of three different species - Clarkia breweri, S. floribunda, and snapdragon, and cDNAs encoding SAMT from these species have been obtained (Negre et al., 2002; Pott et al., 2002; Ross, 2002; Ross et al., 1999). The purified enzymes can methylate both $\mathrm{SA}$ and BA, but all possess $K_{\mathrm{m}}$ values for BA that are 310 -fold higher than their $K_{\mathrm{m}}$-values for SA (Negre et al., 2002; Pott et al., 2002; Ross et al., 1999). In contrast, only one protein with predominantly BA methylating activity has been characterized thus far. The snapdragon BA methyltransferase (BAMT) showed activity with BA but not with $\mathrm{SA}$, although the $K_{\mathrm{m}}$-value for the physiological substrate BA, $1.1 \mathrm{mM}$ (Dudareva et al., 2000), is considerably higher than the reported $K_{\mathrm{m}}$-values for SAMTs using SA, which range from 24 to $83 \mu \mathrm{M}$. So far, no SAMT or BAMT enzymatic activities in these species have been detected in organs other than flowers (a putative SAMT, with a poorly characterized substrate specificity, was reported to be induced in hairy root cultures of Atropa belladonna upon SA treatment; Fukami et al. (2002)). Specifically, neither the expression of SAMT and BAMT could be detected in leaves under normal conditions, nor was the expression of SAMT and $B A M T$ induced in leaves by various treatments, including administration of SA or methyljasmonate (MeJA; Negre et al., 2002; Ross, 2002).

SAMT and BAMT belong to a structurally related group of methyltransferases termed the SABATH family based on the first three identified enzymes belonging to this group, SAMT, BAMT, and theobromine synthase (D'Auria et al., 2003). The SABATH proteins show no significant sequence similarity to other known methyltransferases (D'Auria et al., 2003; Noel et al., 2003). The A. thaliana genome contains 24 intact genes of this type, which are collectively called the AtSABATH genes (D'Auria et al., 2003). Only one of these has been functionally characterized - jasmonic acid carboxyl methyltransferase (JMT; renamed here AtJMT1), the gene encoding the enzyme that catalyzes the formation of MeJA from jasmonic acid (JA)(Seo et al., 2001). Analysis of sequence similarity indicates that none of the 23 uncharacterized AtSABATH proteins is particularly similar in sequence to known SAMTs (i.e. they are all $<45 \%$ identical to the $C$. breweri SAMT) (D'Auria et al., 2003).

We have undertaken the task to determine the function of all AtSABATH genes. As it was previously reported that
A. thaliana could synthesize and emit the ubiquitous plant compound MeSA (Van Poecke et al., 2001), we asked whether the enzyme responsible for methylation of SA belongs to the SABATH protein family of methyltransferases, and if so, in what tissues and under what conditions is its gene expressed? Here, we report the identification of a SABATH gene from $A$. thaliana and its ortholog from $A$. lyrata that encode enzymes embodied with both SAMT and BAMT activities. We also present data suggesting that the $A$. thaliana gene is involved in the plant response to biotic and abiotic stresses.

\section{Results}

\section{Detection of MeSA emission from A. thaliana plants}

We have recently shown that $A$. thaliana plants grown under normal conditions do not emit detectable amounts of benzenoid esters from their vegetative or reproductive tissues (Chen et al., 2003). Similar results had been reported by others (Van Poecke et al., 2001). On the other hand, plants under attack by Pieris rapae caterpillars have been reported to emit a volatile cocktail that includes MeSA (Van Poecke et al., 2001).

We tested several treatments under which biosynthesis and emission of benzenoid esters might be induced. The most effective of the methods we used in inducing MeSA emission was the treatment of detached $A$. thaliana leaves with alamethicin, a voltage-gated ion-channel-forming antibiotic from the fungus Trichoderma viride that had been found to be an efficient inducer of MeSA emission in lima beans (Engelbert et al., 2001). This treatment resulted in MeSA emission levels of $6.4 \pm 0.7 \mathrm{ng} \mathrm{g}^{-1} \mathrm{FW} \mathrm{h}^{-1}$ for several hours after the first $21 \mathrm{~h}$ of exposure to alamethicin (Figure 1a). No MeBA emission was detected following alamethicin treatment, and detached leaves treated with water emitted only trace amounts of MeSA (Figure 1a).

Feeding by the larvae of the diamondback moth (Plutella xylostella) on rosette leaves of intact plants resulted in MeSA emission levels of $0.7 \pm 0.3 \mathrm{ng} \mathrm{g}^{-1}$ $\mathrm{FW} \mathrm{h}^{-1} 21-30 \mathrm{~h}$ after the start of herbivory (Figure $1 \mathrm{~b}$ ). Additionally, small levels of MeBA emission, approximately 10 -fold less than the MeSA levels and at the limit of detection, were also observed (Figure 1b). Treatments with MeJA, physical wounding, and thrip herbivory did not result in detectable levels of either benzenoid ester emission or intracellular concentrations of these compounds. Serendipitously, it was found that uprooting the plant from the soil (which simulates soil disturbance) and placing it in a beaker with its roots submerged in water resulted in emission of both MeSA and MeBA, but the emission levels were at the threshold of detection and therefore difficult to quantify. 

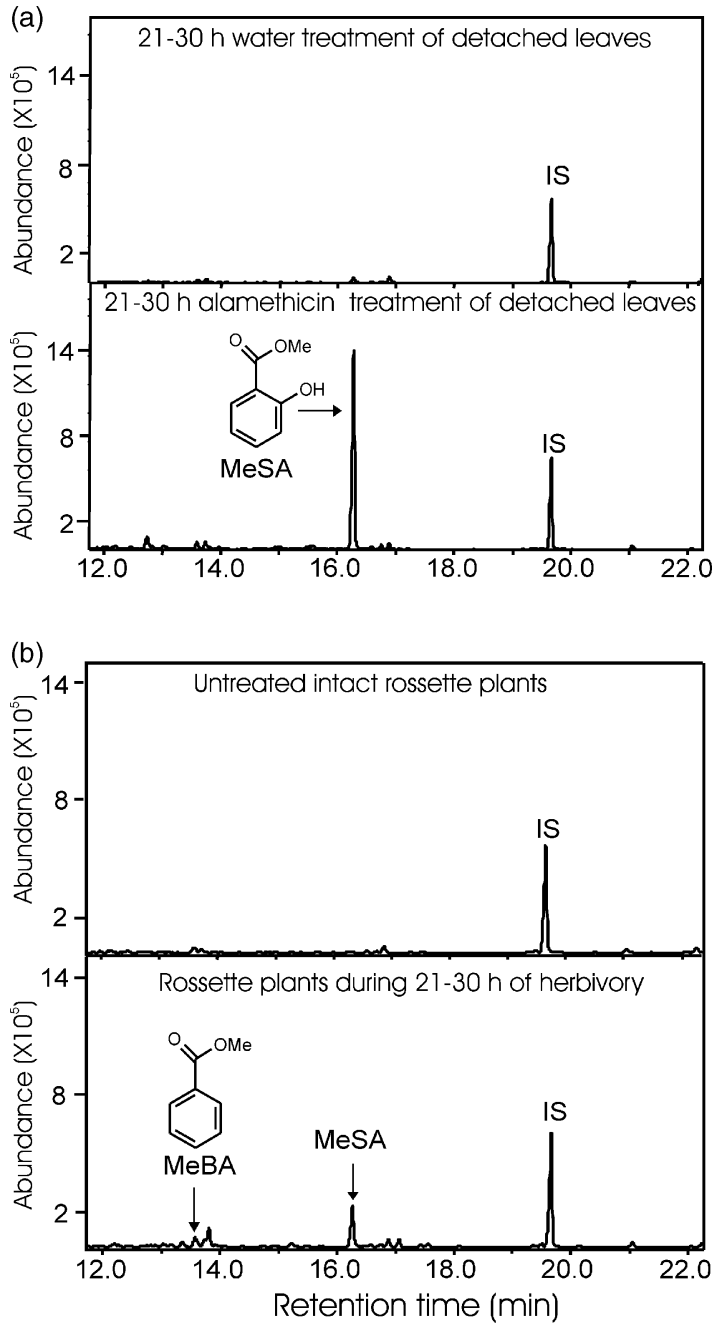

Figure 1. Induction of MeSA and MeBA emission.

Volatiles emitted from (a) detached rosette leaves treated with alamethicin or water, and from (b) whole rosette plants under attack by Plutella xylostella larvae or control (no $P$. xylostella larvae) were collected during $9 \mathrm{~h}$ of closedloop stripping (Donath and Boland, 1995) 21-30 h after the beginning of treatment and identified using GC/MS analysis. Each experiment was repeated three times, and quantitative data were obtained by integration of peaks and comparison with standards. A portion (from 12 to $22 \mathrm{~min}$ ) of one gas chromatogram from each experiment is shown. IS represents the internal standard (nonyl acetate).

Isolation of AtSABATH $c D N A$ from $\mathrm{A}$. thaliana encoding a protein with BAMT and SAMT activities by correlating AtSABATH gene expression with MeSA emission

We had previously constructed 20 pairs of AtSABATH genespecific primers and two pairs of primers that are each specific to two AtSABATH genes (Table 1) to measure the expression of all the 24 AtSABATH genes in various organs (D'Auria et al., 2003). Here, we used these primers in RTPCR experiments (Figure 2) to determine which of the 24 AtSABATH genes were expressed following alamethicin application to detached leaves (Figure 2a, bottom panel)
Table 1 Primers for RT-PCR analysis

\begin{tabular}{|c|c|}
\hline Gene name & Sequences $\left(5^{\prime}\right.$ to $\left.3^{\prime}\right)$ \\
\hline $\begin{array}{r}\text { At5g37970 + } \\
\text { At5g37990 }\end{array}$ & $\begin{array}{l}\text { F: AATGCCAACATTTCCTCAATCGT } \\
\text { R: CCATAAGACAATCTCCAATCATATCG }\end{array}$ \\
\hline At5g38780 & $\begin{array}{l}\text { F: AATGTCAACATCTTCTCAGTCGT } \\
\text { R: CTCAATATTCACTTTCGTTTAAG }\end{array}$ \\
\hline At5g38100 & $\begin{array}{l}\text { F: AATGTCAACATCTTCTCACATGT } \\
\text { R: GTATTTATTTTCGTTTAAGTACTA }\end{array}$ \\
\hline $\begin{array}{r}\text { At1g66690 + } \\
\text { At1g66700 }\end{array}$ & $\begin{array}{l}\text { F: CCATATGACTACTACTCCAGATTGG } \\
\text { R: TGGATCCCGGTTTAGTTCTTGCGAAG }\end{array}$ \\
\hline At3g44840 & $\begin{array}{l}\text { F: ATGATCGGCGGTGAAGGTCC } \\
\text { R: TCAAATTCTTTTAAGCACAATAAGGTAT }\end{array}$ \\
\hline At3g44860 & $\begin{array}{l}\text { F: GATCAATGAAGCCATCTCCACG } \\
\text { R: CTTGCGTCTTCTCTTTCACAAGC }\end{array}$ \\
\hline At3g44870 & $\begin{array}{l}\text { F: ATCGGTGGTGAAGGTCCCAAC } \\
\text { R: CTTTGGCTATCTGAGGAAGCA }\end{array}$ \\
\hline At1g68040 & $\begin{array}{l}\text { F: AATGGGAAAAGTGGAGATTGTT } \\
\text { R: CAGCCAAAAACTTGTCAAATA }\end{array}$ \\
\hline At1g15125 & $\begin{array}{l}\text { F: AATGAACGGTGGAGACGGT } \\
\text { R: CATATATCAGATGTCATGACAG }\end{array}$ \\
\hline At5g55250 & $\begin{array}{l}\text { F: AATGGGTTCTAAGGGAGCAAC } \\
\text { R: ATTAAGTAAAAGACAAAGAAGCGAC }\end{array}$ \\
\hline At5g56300 & $\begin{array}{l}\text { F: AATGACGGCCAAAGACTGGA } \\
\text { R: TTAAACTCGGATGGCGGAAAAC }\end{array}$ \\
\hline At4g26420 & $\begin{array}{l}\text { F: AATGGAGTCGTCACGGAGC } \\
\text { R: ACTACGCATGTTATGTACATA }\end{array}$ \\
\hline At1g19640 & $\begin{array}{l}\text { F: AATGGAGGTAATGCGAGTTCTT } \\
\text { R: TCAACCGGCTCTAACGAGCGA }\end{array}$ \\
\hline At2g14060 & $\begin{array}{l}\text { F: AATGGACTCTTTGGTTGGACGCT } \\
\text { R: CAATGAACTTTATTTCCTAGTC }\end{array}$ \\
\hline At3g21950 & $\begin{array}{l}\text { F: CATCAAAGTGGCTGACTTGGGAT } \\
\text { R: AACGAAACAAAGAAATTAACGGTTG }\end{array}$ \\
\hline At5g38020 & $\begin{array}{l}\text { F: AATGGGTGTTCTAAGCATGAAG } \\
\text { R: GTTACTTCCTTGTCAAGGAAAC }\end{array}$ \\
\hline At5g66430 & $\begin{array}{l}\text { F: AATGTACAGTTTTGTGAATGCTC } \\
\text { R: GACAGATTACTTTCGTATCAAAG }\end{array}$ \\
\hline At3g11480 & $\begin{array}{l}\text { F: AATGGTATATATGTTGACCAA } \\
\text { R: CTTCTTACTTCTTAGTCAAGGA }\end{array}$ \\
\hline At4g36470 & $\begin{array}{l}\text { F: AATGGATAAGAAGGATATGGAG } \\
\text { R: ATCAGAGCTTCTTTCTTAGGAC }\end{array}$ \\
\hline At1g66720 & $\begin{array}{l}\text { F: AATGTCACCTACTCCAGAATGGGT } \\
\text { R: TCAGTTCTTGCGAAGTACGATGAG }\end{array}$ \\
\hline At5g04370 & $\begin{array}{l}\text { F: AATGGATTCAAGATTCACCGATTC } \\
\text { R: CGCCAAGATTAAACTTTAGGACATGA }\end{array}$ \\
\hline At5g04380 & $\begin{array}{l}\text { F: AGTGAGTATAAAGTGGATGAAGAGAGAAGT } \\
\text { R: TTATTTCCGAACCAGTGAAACTATTAGAG }\end{array}$ \\
\hline
\end{tabular}

$F$, forward primer; $R$, reverse primer. 


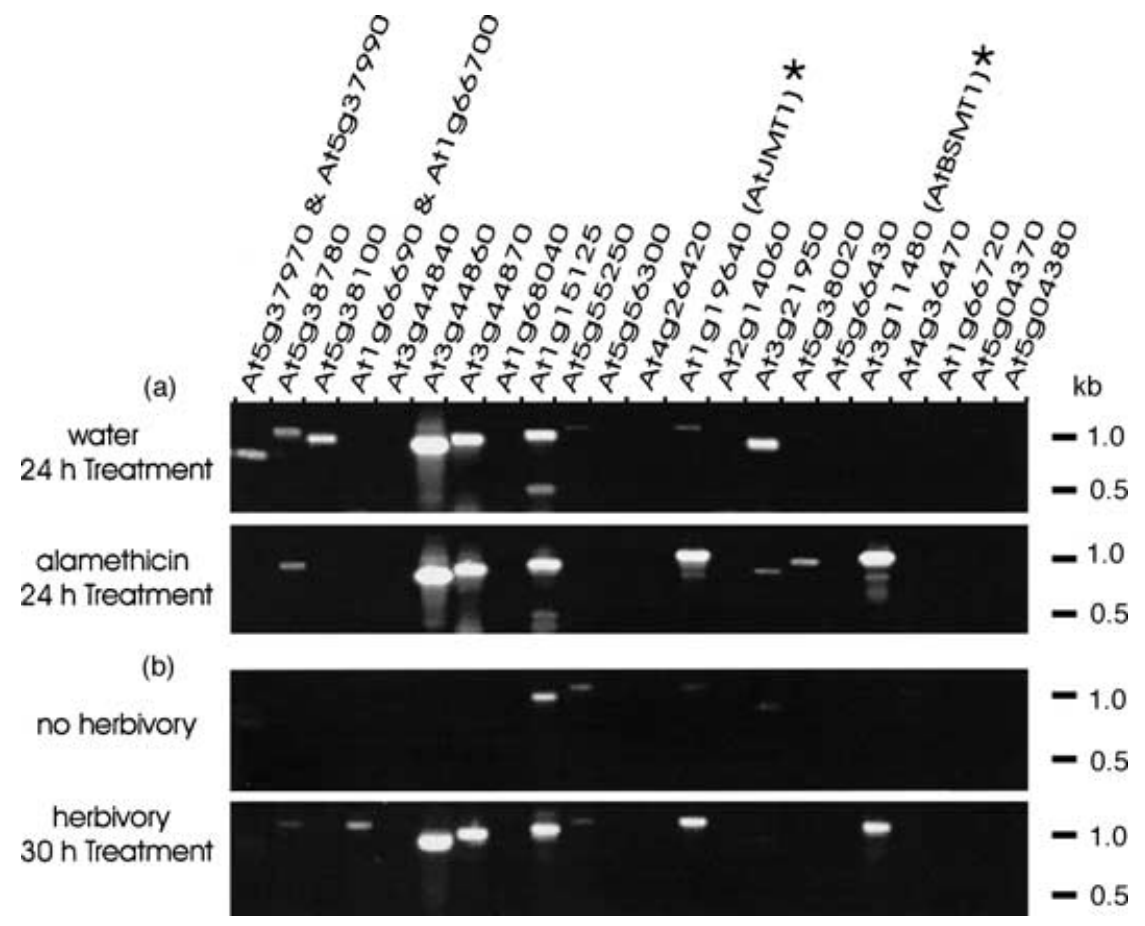

Figure 2. RT-PCR analysis of transcript levels of individual AtSABATH genes.

(a) AtSABATH gene expression in detached rosette leaves treated for $24 \mathrm{~h}$ with water only (top panel) and with alamethicin (bottom panel). (b) AtSABATH gene expression in intact rosette leaves from plants not attacked by $P$. xylostella larvae (top panel) and in attached rosette leaves damaged by $P$. xylostella larvae. The expression of the 24 AtSABATH genes was examined in 22 RT-PCR reactions. Because of high sequence similarity of At5g37970 and At5g37990, and At1g66690 and At1g66700, PCR products obtained from one or the other member of each of these two pairs of genes could not be distinguished. RT-PCR with primers for $\beta$-tubulin and actin- 8 was used to judge equality of the concentration of the cDNA templates used in each treatment-control combination, and loaded samples were normalized accordingly, loaded and separated on a $1 \%$ agarose gel. The two genes whose transcripts levels are high in both alamethicin- and P.xylostella-treated leaves and low in both controls are indicated with asterisks.

and $P$. xylostella herbivory on attached leaves (Figure 2b, bottom panel), two treatments that induce MeSA emission, but were not expressed in the respective control treatments, which did not result in MeSA emission. Intact, undamaged leaves showed the fewest number of AtSABATH genes expressed (Figure 2b, top panel). Detached leaves treated with water only (Figure $2 a$, top panel) showed the expression of several additional AtSABATH genes, some of them also induced by $P$. xylostella herbivory and alamethicin application (e.g. At3g44860 and At3g44870), suggesting that the function of these genes is in a general response to stress. One gene, At5g38020, was induced by alamethicin but not by herbivory, and one or the other member of the closely related pair of genes At1g66690 and At1g66700 (which encode proteins that are $92 \%$ identical to each other) was induced by herbivory but not by alamethicin.

However, the expression pattern of only two genes, At1g19640 and At3g11480, correlated perfectly with MeSA emission: their steady-state mRNA levels increased substantially following both alamethicin application and $P$. xylostella herbivory, the two MeSA emission-inducing treatments, in comparison to both control treatments that did not result in MeSA emission (Figures 1 and 2). One of these two induced genes, At1g19640, had previously been identified as AtJMT1 (Seo et al., 2001). The full-length cDNA clone of the remaining induced gene, At3g11480, was obtained by RT-PCR, expressed in Escherichia coli, and the crude $E$. coli protein extract was tested for enzymatic activity using several putative substrates, including $\mathrm{SA}$ and BA. The lysate of $E$. coli cells expressing gene
At3g11480 showed activity with BA and SA (see below), and this gene was therefore named AtBSMT1. Proteins obtained in $E$. coli from the expression of full-length cDNA clones of, among others, At1g66700 and At1g15125 did not have methylating activities with SA or BA.

\section{Additional characterization of AtBSMT1 expression}

To determine the tissues in which AtBSMT1 is expressed, total RNA was isolated from flowers, leaves, stems, siliques, and roots of $A$. thaliana. These RNA preparations were used in RNA gel blot analysis. The highest levels of AtBSMT1 transcripts were observed in flowers, lower levels were seen in leaves and stems, and little or no AtBSMT1 transcripts were observed in roots or siliques of plants grown under standard conditions (Figure 3a).

Further experiments were performed to identify conditions under which AtBSMT1 gene expression is induced in $A$. thaliana leaves. Leaf wounding and uprooting caused AtBSMT1 transcripts to accumulate quickly within $2 \mathrm{~h}$ after treatment (with uprooting being more effective), and transcript levels declined rapidly within $24 \mathrm{~h}$ (Figure $3 \mathrm{~b}$ ). In contrast, MeJA treatment, in which plants were exposed to MeJA vapors in a closed container, resulted in slower induction, and transcript levels at $24 \mathrm{~h}$ after treatment were higher than those at $2 \mathrm{~h}$ after the beginning of induction. $\beta$ Glucuronidase (GUS) staining of several lines of transgenic plants carrying an AtBSMT1 promoter-GUS fusion gene indicated that under normal growing conditions, the AtBSMT1 promoter was active in leaves only at the base of the trichomes, in the hydathodes (Figure $4 a, b$ ), and in the 
Figure 3. Expression analysis of AtBSMT1 (a) RNA gel blot analysis of the relative abundance of AtBSMT1 mRNA transcripts in different organs. Top, a representative gel blot of RNA samples from flowers (F), leaves (L), siliques $(\mathrm{Si})$, stems $(\mathrm{St})$, and roots (R). Bottom, a graphical representation of the results representing the average of two independent experiments. Tissues were harvested from mature, bolted plants, and leaf samples came from the rosette leaves.

(b) Analysis of AtBSMT1 gene expression in $A$. thaliana rosette leaves under various treatments. Top, a representative gel blot. Bottom, a graphical representation of the normalized results representing the average of two independent experiments. Leaves were harvested from rosette plants. Lanes were loaded with $10 \mu \mathrm{g}$ of total RNA. After probing with the AtBSMT1 probe and quantification of the results, the blot was stripped and re-probed with an $18 \mathrm{~S}$ rDNA probe to normalize samples. (a)

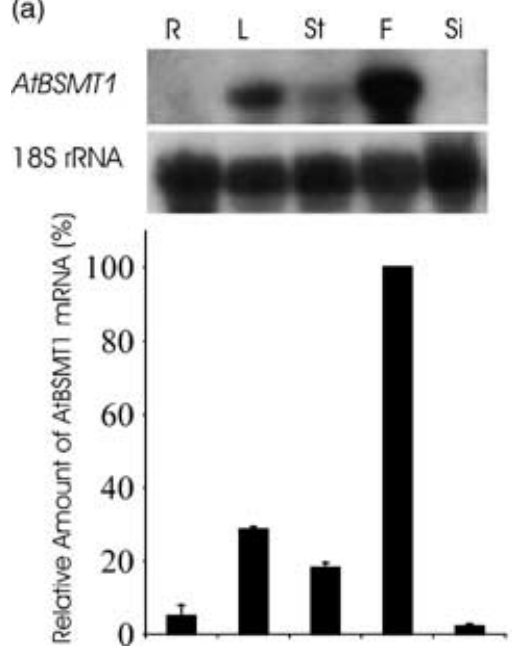

(b)
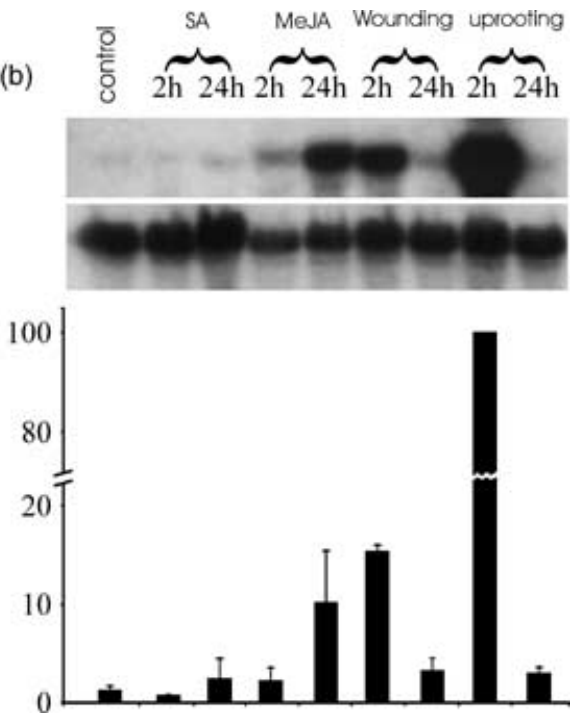

upper part of the sepals of flowers (Figure 4c). In addition, strong AtBSMT1 promoter activity was observed around lesions in the leaves caused by thrips of the genus Frankliniella (Figure 4d). These herbivores damage the plant by sucking the cytoplasm out of the cell with their specialized proboscis, leaving the cell walls intact (Hunter and Ullman, 1992).

Isolation of the A. lyrata ortholog of AtSAMT1 and characterization of its expression

We isolated a cDNA of the orthologous gene to AtSAMT1 from the closely related perennial species $A$. lyrata by

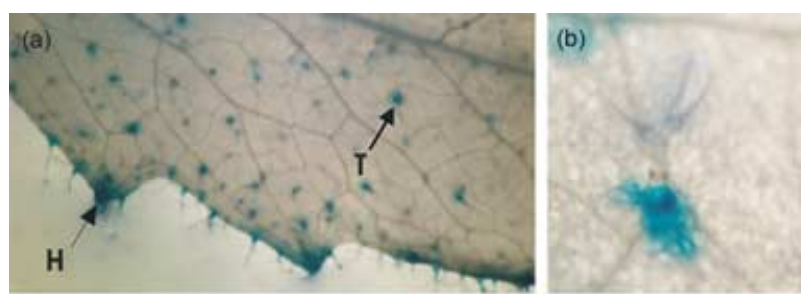

(c)

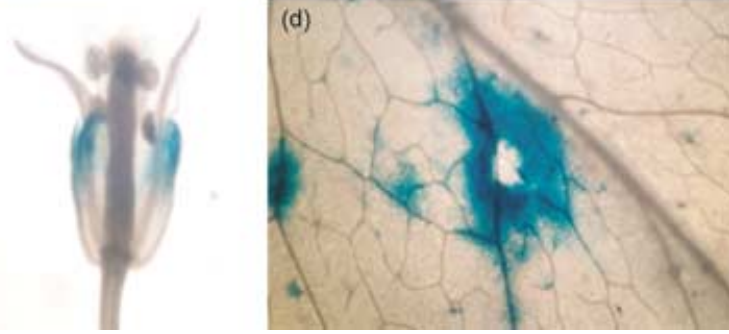

Figure 4. AtSAMT1 promoter::GUS fusion analysis.

(a) Leaf area, showing staining at the base of trichomes (T) and at the hydathodes $(\mathrm{H})$, (b) close-up of a trichome, (c) staining of flowers showing activity in the sepals, and (d) close-up of a leaf area damaged by thrips, showing induction of AtSAMT1 in the area surrounding the damage.
RT-PCR with leaf mRNA and the AtBSMT1 primer pair. The A. lyrata cDNA was expressed in E. coli, and the resulting protein also showed methyltransferae activity with SA and BA (see below). It was therefore named AIBSMT1.

To verify that the $A$. lyrata gene AIBSMT1 is orthologous to AtBSMT1, we performed PCR on genomic DNA from both $A$. thaliana and $A$. lyrata. Two primers were designed based on the genomic sequence of $A$. thaliana. The forward primer 5'-GGTTCTCACCTTCATCGGAA-3' corresponds to exon 4 of At3g11480 (AtBSMT1), and the reverse primer 5'-CTGTCTACTGCTGCTCTGATC-3' corresponds to exon 5 of gene At3g11490, the adjacent downstream gene to At3g11480 (which is transcribed in the direction toward At3g11480) on chromosome III (Figure 5). When A. thaliana genomic DNA was used in the PCR reaction, a fragment of 1292 nucleotides was obtained (Figure 5). When A. lyrata genomic DNA was used, a fragment of 1256 nucleotides was amplified, which showed $82 \%$ identity to the $A$. thaliana fragment (Figure 5). Sequence analysis confirmed that one end of the $A$. lyrata fragment comprises the sequence of the $3^{\prime}$ end of $A I B S M T 1$, including part of the fourth exon, an intron, and the fifth exon. The sequence identity of this segment to the corresponding $A$. thaliana fragment is $94 \%$ in the exons and $75 \%$ in the intron (there are also three deletions/insertions greater than one nucleotide each in one sequence compared with the other in this area). The other end of the fragment, containing the $3^{\prime}$ end of the coding region of the adjacent gene, is $93 \%$ identical to the $3^{\prime}$-coding region of At3g11490. The middle of this fragment constitutes the intergenic spacer, which has $84 \%$ sequence conservation between the two genomes (but 10 deletions/insertions). As these results indicate that the same gene is found downstream to AtBSMT1 and AIBSMT1, we conclude that the latter two are orthologous. 
At $3 g 11480$ exon 4

Atg GGTTCTCACCTTCATCGGAAGAAACACTCTTAACGATCCATTGTATAGAGATTGTTGTCACTTTTGGACATTGCTATCAAACTCTCTCCGTGACCTAGTCTTTGAGGTAT 110 AlG GGTTCTCACCTTCATCGGAAGAAACACTCTGAACGATCCATTGTATAGAGATTGTTGTCACTTTTGGACATTGCTATCAAAATCTCTCCGCGACCTAGTCTTCGAGGTAT 110

At 3g11480 intron 4

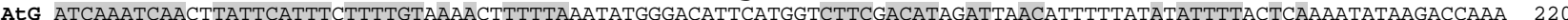

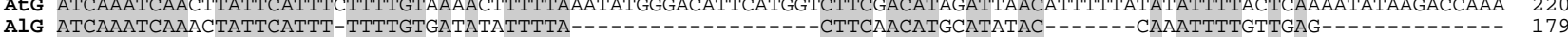

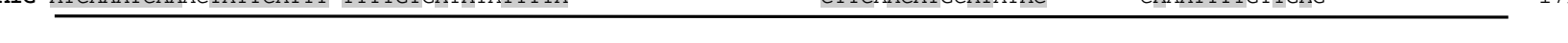

Atg CATATATATtATtgTgGAACATGGTACTCATtGCTATCTTCATATAAA-CAGGGTCTTGTGAgTGAgTCAAAGCTGGACGCATTCAACATGCCGTTTTATGATCCGAACG 329 AlG - -

Atg TACAAGAACTCAAAGAAGTGATACAAAAAGAGGGCTCTTTTGAAATCAATGAATTGGAGTCACATGGATTTGATCTTGGTCACT---ACTACGAAGAAGATGACTTTGAA 436 Alg TACAAGAACTCAAACAAGTGATACGAAACGAGGGCTCTTTTGAAATCAATGAATTGGAGACGCATGGATTCGATCTTGGTCATAGTAACTACGAAGAAGATGACTATGAA 387

At $3 g 11480$ exon 5

Atg GCAGGACGCAATGAAGCTAATGGCATAAGAGCTGTTAGTGAACCAATGCTCATTGCTCATTTTGGAGAAGAAATTATCGATACCTTGTTCGATAAGTATGCATACCATGT 546 AlG GCAGGACACGATGAAGCCAATTGTATAAGAGCTGTTAGTGAACCAATGCTCGTTGCTCATTTTGGAGAAGACATTATCGATACCTTGTTCGATAAGTATGCACACCATGT 497

Atg GACTCAACATGCCAACTGCAGGAACAAAACGACTGTCAGTCTTGTCGTTTCCTTGACTAAGAAGTAAGAAGTAATCAACTTCTGTCATGTTGCTCTATTTGTATTTATTT 656 AlG GACTCAGCATGCCAACTGCAGGAACAAAACGACTGTCAGTCTTGTCGTTTCGTTGACTAAGA-------AATAATCAACTTCTGTCGTAT------GTTTTTTCTTTATT 594

Atg ACTACTGTTATTTTGTTTCCTTGAATAAATtTCAACACCTGCCATTCAATGTGATGATGTGGAgCTAGTATTGGAAAAAAGTGTGACTGTAATATATTATGgTAAATTTG 766 Alg ACTACTGTTCATTTGTTTCCTTTAATAAATTCCAAGATCTGCCACTCAATGTGGTG--------CTAGTATTGGAAAAAAGTGTGATTGTAATGTGTTTTGTTCTGTTCG 696

\section{intergenic region}

AtG GT----AAATGATTTATTT---------CCTGCAAAAGTT--GCAGATA---------CTGC-----TTTCAAGTATGAAGATAAATCCTTGTGAAGAGAAAGAGAAACT 847 Alg ATTTCAACATCTTTTGTTAATAACCAAATCCGTAACGATTTGGTAAATGAGCTATTTCCTGCAAAACTTTCAAGTACCAAGATAAAACCTTAAGAAGAGAAACAGACACT 806

AtG GAGAAACTTATCATTGACATTATATGCAGAAACGTAGCATTTCTTGATACACAAGCAACCATCATCAAAATTCAAAAGCATC----CATCCATCCCACAAAACCCAAATT 953 AlG ----- TATCATTGACATTATATGCAGAAACGTAGCATTTCTTGATACACAAGCAACCATCATCAAAATTCAAAAGCATCTATCCATCCATCCCACAAAACCCGAATC 908

Atg AAGAAA-TCCAAAAGCTGGATCAAAAACATCAACAGT-AACCTTT-TTTCATCGCCAAGCTTCGAATAGCTCAACTCTGTAATTCACACGTCCCACAATACTACTCGCTT 1060 AlG AAGAAAATCCAAAAGATGGATCAAAAACATCAACAATTAACCTTTCTIT CCGCCAAGCTTCGAATAGCTCAACTCTGTAGTTCACACGTCCCACAATACTACTCGCTT 1018

Atg TCGACATGGTTCTCTGCTCCTCCCAACCAATGTGTTTCTGTCCAAAACCTCCACTGCTGTTATTAACCAGAGAAGACTTTTTTATGTCAACAAGTTCTACAGATTCGGCA 1170 Alg TCGACATGGTTCTCTGCTCCTCCCAACCATTGTGCTTCTGTCCAAAACCTCCATTGTTGCTGTTAACCAGAGAAGAATTTTTATGTCAACAAGTTCTCGAGATTCCACA 1128

\section{At $3 g 11490$ exon 5}

Atg CATTCTTCTTCTTCTTC------ATCTGCAGACTCTTCTTTGTCTTTCATCTCCGCCTCAAAGTTGTCTAAAGTTTCCTCCTTGTTAGCTTTCATGAGATGTAACAACTG 1274 Alg CATTCTTCTTCTTCTTCTTCTTCATCTGCAGACTCTTCTTTGTCTTTCATCTCTGCCTCAAAGTTGTCTAAACTTTCCTCCTTGTTAGCTTTCATGAGATGCAACAACTG 1238

Atg TCTACTGCTGCTCTGATC 1292

AlG TCTACTGCTGCTCTGATC 1256

Figure 5. Sequence and structures of a segment of the $A$. thaliana genome (AtG) that contains the $3^{\prime}$ end of the AtBSMT1 (At3g11480) gene and the $3^{\prime}$ end of At3g11490, and the corresponding A. lyrata genomic segment (AIG).

The intron in BSMT1 and the intergenic region are underlined. The stop codon of each gene is shown in white letters on black background. Identical nucleotides in a given position are shaded.

RNA gel blot analysis showed that AIBSMT1 transcript levels were, similarly to AtBSMT1 transcripts in A. thaliana, higher in flowers and detectable also in leaves (Figure 6).

Analysis of the sequences of the proteins encoded by AtBSMT1 and AIBSMT1

The AtBSMT1 encodes a protein comprising 379 amino acid residues, while the AIBSMT1-encoded protein is one residue longer (Figure 7a). The two proteins are $92 \%$ identical, and the critical residues putatively involved in binding the acid substrate are identical in both (Figure 7a). Both AtBSMT1 and AIBSMT1 are less than $50 \%$ identical to any of the four currently known SAMTs or the snapdragon BAMT (AmBAMT). Compared with most of these other proteins, both AtBSMT1 and AIBSMT1 possess an N-terminal extension of about 20 amino acids. The snapdragon SAMT (AmSAMT) also has an N-terminal extension, but it is shorter (Figure 7a). Phylogenetic analysis of the sequences presented in Figure 7(a) indicates that AtBSMT1 and AIBSMT1 are more similar to AmBAMT and also to AtJMT1 than they are to other SAMTs, including $C$. breweri SAMT (CbSAMT; Figure 7b).

\section{Enzymatic characterization of AtBSMT1 and AIBSMT1}

The coding regions of AtBSMT1 and AIBSMT1 were ligated into the vector $\mathrm{PCRT7/CT-TOPO} \mathrm{TA} \mathrm{for} \mathrm{functional} \mathrm{expres-}$ sion in E. coli as non-fusion proteins. The two proteins were purified to near homogeneity in two steps, first using anion-exchange column diethylaminoethyl cellulose followed by Mono- $Q$ anion-exchange chromatography (Figure 8; Table 2). The molecular mass of both holoenzymes was estimated by size exclusion chromatography to be $91 \mathrm{kDa}$. The subunit molecular mass of both enzymes, calculated from their sequences, is $43.4 \mathrm{kDa}$ (Figure 8). 


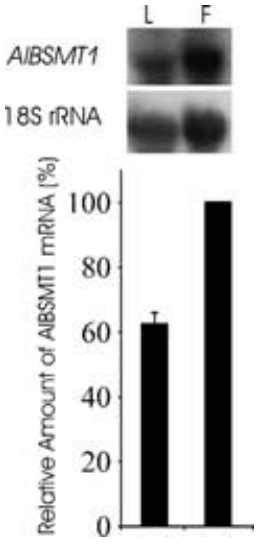

Figure 6. RNA gel blot analysis of the relative abundance of AIBSMT1 mRNA transcripts.

Top, a representative gel blot of RNA samples from flowers (F) and leaves (L). Bottom, a graphical representation of the results representing the average of two independent experiments. Tissues were harvested from flowering plants. Lanes were loaded with $10 \mu \mathrm{g}$ of total RNA. After probing with the AtBSMT1 probe and quantification of the results, the blot was stripped and re-probed with an $18 \mathrm{~S}$ rDNA probe to normalize samples.

Together, these results indicate that the AtBSMT1 and AIBSMT1 enzymes exist as homodimers under physiological conditions, as do previously characterized SAMTs and BAMTs (Murfitt et al., 2000; Ross et al., 1999).

The two enzymes were further evaluated for their ability to catalyze the SAM-dependent carboxyl methylation of a number of potential substrates (Table 3 ). Of the substrates tested, SA, BA, anthranilic acid, and $m$-hydroxybenzoic acid all served as in vitro substrates in the reactions catalyzed by the AtBSMT1 protein. At substrate concentrations of $1 \mathrm{mM}$, AtBSMT1 showed the highest activity with BA and $m$ hydroxybenzoic acid rather than with SA. AIBSMT1 also exhibited methyltransferase activity with BA, SA, and anthranilic acid, but lacked detectable activity with 3-hydroxybenzoic acid. Notably, AIBSMT1 also exhibited a higher activity with BA than with SA under the conditions tested.

Steady-state kinetic parameters for each enzyme were also determined (Table 2). AtBSMT1 and AIBSMT1 showed

Figure 7. Sequence analyses of AtBSMT1, AIBSMT1, and other known SABATH proteins.

(a) Amino acid sequence alignment of all proteins using the CLUSTALX program (Thompson et al., 1997). Amino acid residues conserved in five or more sequences are shown in white letters on black background. The positions of the residues involved in the acid substrate binding site, identified from the three-dimensional structure of CbSAMT (Noel et al., 2003; Zubieta et al., 2003) are indicated with the symbol \#.

(b) Maximum parsimony phylogenetic tree constructed from the alignment in (a) using the program PAUP*.

CbSAMT: $C$. breweriSAMT (Accession \# AF133053); AmSAMT, Antirrhinum majus (snapdragon) SAMT (Accession \# AF515284); SfSAMT, S. floribunda SAMT (Accession \# AF308570); AmBAMT: A. majus BAMT (Accession \# AF198492); AtJMT1, A. thaliana JMT (Accession \# AY008434); AbSAMT, A. belladonna SAMT (Accession \# AB049752). The Accession number of AtBSMT1 is AY224595, and the Accession number of AIBSMT1 is AY224596. similar apparent $K_{\mathrm{m}}$-values for SAM, $67 \mu \mathrm{M}$ and $87 \mu \mathrm{M}$, respectively, and a twofold difference in apparent $K_{\mathrm{m}}$ values for BA, 65 and $131 \mu \mathrm{M}$, respectively. However, the two enzymes had very different $K_{\mathrm{m}}$-values for SA: the

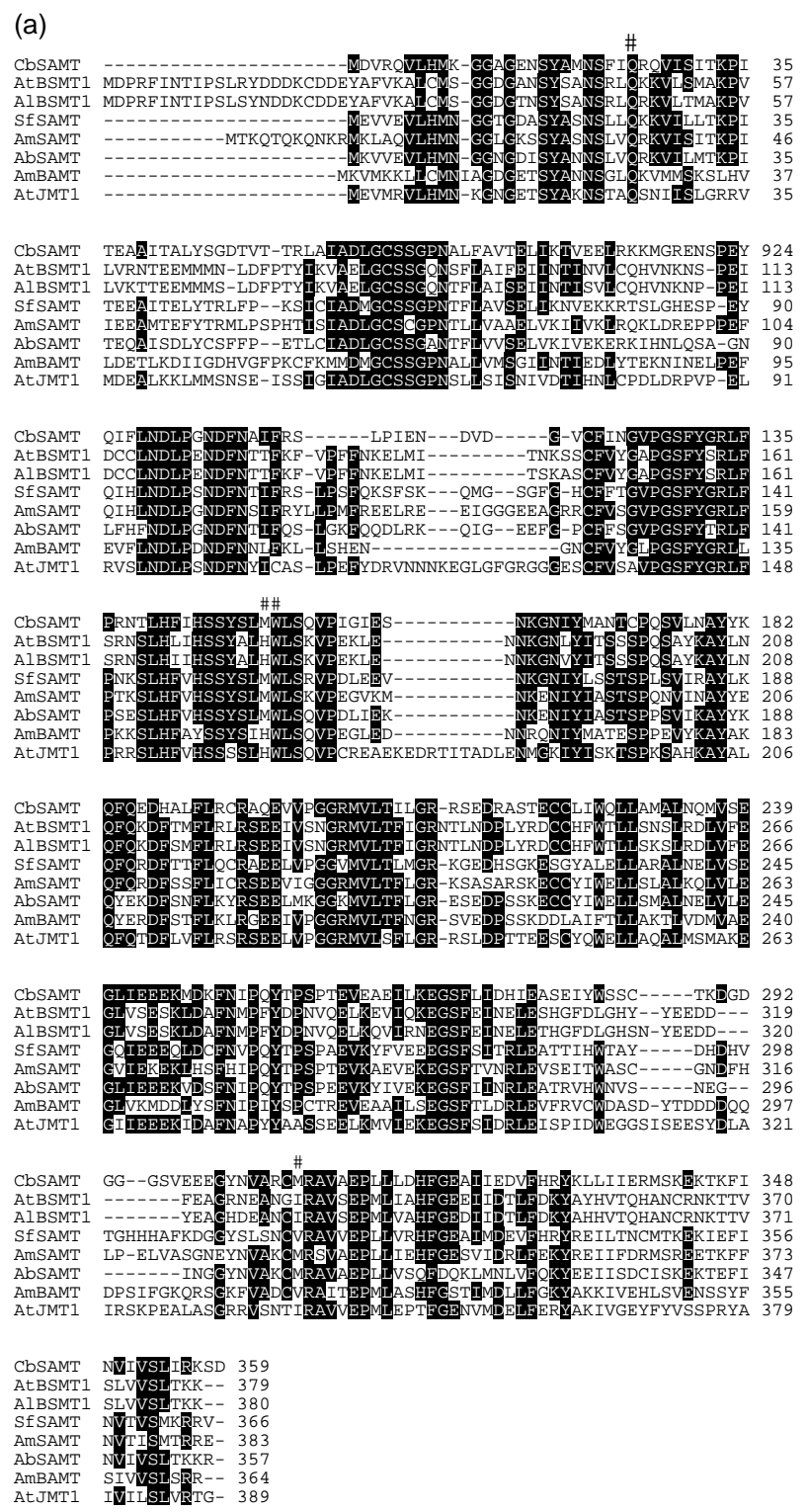

(b)

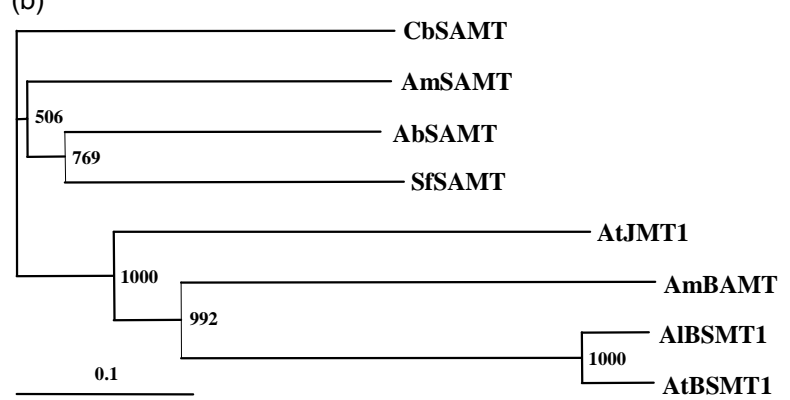




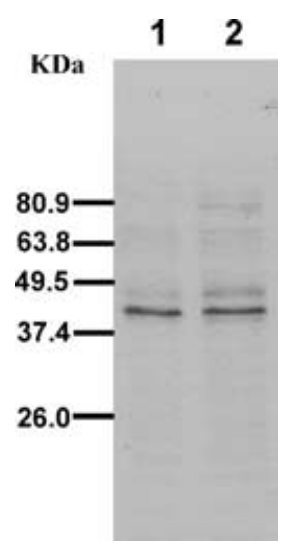

Figure 8. Purified AtBSMT1 (lane 1) and AIBSMT1 (lane 2). An aliquot $(1 \mu \mathrm{g})$ of each enzyme from the Mono- $Q$ fraction with the highest level of BAMT and SAMT activities was electrophoresed on a $10 \%$ SDSPAGE. The gel was stained with Coomassie Bright Blue. The positions of protein molecular weight markers are indicated on the left.

$K_{\mathrm{m}}$-value of AtBSMT1 for SA was $16 \mu \mathrm{M}$, whereas the $K_{\mathrm{m}}$ value of AIBSMT1 for SA was $127 \mu \mathrm{M}$ (Table 2). Thus, while the $A$. thaliana enzyme had similar catalytic efficiency with both SA and BA, the $A$. lyrata enzyme was about threefold more efficient with BA than AtBSMT1, but about 11-fold less efficient SA than with the $A$. thaliana enzyme. Put another way, the catalytic efficiency of AtBSMT1 with SA was $50 \%$ higher than with $B A$, whereas the catalytic efficiency of AIBSMT1 with SA was 24-fold lower than with BA.

\section{Discussion}

\section{Identification of AtBSMT1}

All previously characterized carboxyl methyltransferases involved in the formation of various plant-specialized metabolites belong to the SABATH family (D'Auria et al., 2003), of which CbSAMT was the first recognized member (Ross et al., 1999). A. thaliana contains 24 SABATH family members (D'Auria et al., 2003). However, the identification of $A$. thaliana genes that encode proteins with SAMT or BAMT activity has not been straightforward. The $A$. thaliana
Table 3 Relative activity of AtBSMT1 and AIBSMT1 with benzoic acid, salicylic acid, and related substrates

\begin{tabular}{lcc}
\hline & \multicolumn{2}{c}{ Relative activity $^{\mathrm{a}}(\%)$} \\
\cline { 2 - 3 } Substrate & AtBSMT1 & AlBSMT1 \\
\hline Benzoic acid & 100 & 100 \\
Salicylic acid & 46 & 16 \\
Anthranilic acid & 35 & 32 \\
$m$-Hydroxybenzoic acid & 54 & 0 \\
$p$-Hydroxybenzoic acid & 0 & 0 \\
Vanillic acid & 0 & 0 \\
Caffeic acid & 0 & 0 \\
$p$-Coumaric acid & 0 & 0 \\
Cinnamic acid & 0 & 0 \\
( \pm )Jasmonic acid & 0 & 0 \\
$p$-Aminobenzoic acid & 0 & 0 \\
\hline
\end{tabular}

${ }^{a}$ Values are averages of three independent measurements. All substrates were tested at $1 \mathrm{mM}$ concentration. The activity of the two enzymes with benzoic acid was set arbitrarily at $100 \%$.

SABATH protein with the highest sequence identity to known SAMTs from other species is AtJMT1 (Figure 7b), and this enzyme has been shown to methylate JA but not SA or BA (Seo et al., 2001). In addition, a group of seven AtSABATH proteins cluster together with AmBAMT (D'Auria et al., 2003), but these seven AtSABATH proteins are all equally divergent in primary sequence from AmBAMT (with approximately $40 \%$ identity to AmBAMT). Thus, an identification and functional annotation of $A$. thaliana genes encoding SAMT or BAMT activities could not be straightforwardly attempted based simply on overall sequence similarity.

Instead, we employed a biochemical genomics approach that involved metabolic profiling and the compilation of gene expression data of the entire family, together with enzymatic assays with potential substrates. By correlating the expression of AtSABATH genes under two types of treatments that induce MeSA emission (alamethicin treatment of cut leaves and $P$. xylostella herbivory on rosette plants) and two types of treatments that do not result in MeSA emission (water treatment of cut leaves and normal growth conditions), two strong candidate genes for SAMTencoding capacity were identified. One of these genes, AtJMT1, was ruled out based on previously published work

Table 2 Properties of AtBSMT1 and AIBSMT1

\begin{tabular}{|c|c|c|c|c|c|c|c|c|c|}
\hline Enzyme & $\begin{array}{l}\text { Holoenzyme } \\
\text { mass (Da) by } \\
\text { size exclusion } \\
\text { chromatography }\end{array}$ & $\begin{array}{l}\text { Subunit } \\
\text { mass (Da) by } \\
\text { SDS-PAGE }\end{array}$ & $\begin{array}{l}\text { Calculated } \\
\text { subunit } \\
\text { mass (Da) }\end{array}$ & $\begin{array}{l}\mathrm{pH} \\
\text { optimum }\end{array}$ & $\begin{array}{l}\text { Apparent } \\
K_{\mathrm{m}}(\mu \mathrm{M}) \\
\text { for SAM }\end{array}$ & Substrate & $\begin{array}{l}\text { Apparent } \\
K_{\mathrm{m}}(\mu \mathrm{M}) \\
\text { for acid }\end{array}$ & $K_{\text {cat }}\left(\sec ^{-1}\right)$ & $\begin{array}{l}K_{\text {cat }} / K_{\mathrm{m}} \\
\left(\mathrm{mM}^{-1} \mathrm{sec}^{-1}\right)\end{array}$ \\
\hline \multirow[t]{2}{*}{ AtBSMT1 } & approx. 91000 & approx. 44000 & 43365 & $7-8$ & 67 & Benzoic acid & 65 & 0.19 & 2.9 \\
\hline & & & & & & Salicylic acid & 16 & 0.07 & 4.3 \\
\hline \multirow[t]{2}{*}{ AIBSMT1 } & approx. 91000 & approx. 44000 & 43363 & $7-8$ & 87 & Benzoic acid & 131 & 0.3 & 9.6 \\
\hline & & & & & & Salicylic acid & 127 & 0.05 & 0.4 \\
\hline
\end{tabular}


by Seo et al. (2001), and the second one, At3g11480, was expressed in $E$. coli and shown by biochemical assays to indeed encode an enzyme, AtBSMT1, with SA- as well as BA-methylating activities.

However, until all 24 AtSABATH proteins are thoroughly characterized, it is not possible to conclude that all the A. thaliana genes encoding enzymes with BAMT/SAMT activities have been determined. Nor can it be concluded that all possible substrates of AtBSMT1 have been identified.

\section{Origin, structure, and evolution of the AtBSMT1 and AIBSMT1 proteins}

The sequences of AtBSMT1 and AIBSMT1 are not particularly closely related to other known SAMTs. Instead, they are somewhat more closely related to AmBAMT (Figure 7). In addition, two critical residues important in the binding of SA in SAMTs, Met 150 and Met 308, are replaced in AtBSMT1, AIBSMT1, and AmBAMT, all three having His in the equivalent Met 150 position and Ile, Ile, and $\mathrm{Val}$ in the equivalent Met 308 position, respectively (Figure 7a). However, active site modeling based on the recently determined three-dimensional structure of CbSAMT (Noel et al., 2003; Zubieta et al., 2003) indicates that the overall topology of the active sites of AtBSMT1 and AtBSMT1, which are identical, are more similar to the topology of the active site of SAMTs, while the topology of the active site of AmBAMT is quite distinct (data not shown). In addition, here we have presented data showing that both AtBSMT1 and AIBSMT1 are equally active with BA, but they differ in their catalytic efficiencies with SA, suggesting that the ancestor enzyme possessed a BAMT activity with low levels of SAMT activity, and that AtBSMT1 evolved its higher catalytic activity with SA only after the $A$. thaliana and the $A$. lyrata lineages diverged. These somewhat conflicting data make it difficult to retrace a clear evolutionary path for these two enzymes, emphasizing a common observation regarding enzymes of secondary metabolism whereby one or a few amino acid substitutions may bring about a change in substrate specificity (Frick and Kutchan, 1999; Gang et al., 2002; Wang and Pichersky, 1999). As AtBSMT1 and AIBSMT1 have identical active sites, the difference in their catalytic efficiency with SA must therefore be due to of small changes in the structure of the protein outside the active site.

The presence of an ortholog of the AtBSMT1 in A. lyrata, a close relative of $A$. thaliana whose lineage diverged 5 million years ago (Koch et al., 2000), presents a unique opportunity to address these questions and to examine the evolution of substrate specificity in the SABATH family. It would also be interesting to examine whether A. lyrata plants emit mostly MeBA or MeSA in response to conditions that result in mostly MeSA emission in A. thaliana.
The involvement of AtBSMT1 in the response to biotic and abiotic stresses

The specific physiological role of AtBSMT1 is not yet clear. Under normal conditions, AtBSMT1 is most highly expressed in flowers, and its expression in leaves is restricted specifically to trichomes and hydathodes. However, headspace analysis of $A$. thaliana leaves and flowers has failed to detect emission of benzenoid esters from either organs under normal growth conditions (Chen et al., 2003), and internal levels of such esters were also below our detection limits (Chen and Pichersky, unpublished).

Certain biotic and abiotic challenges to leaves led to the induction of $A t B S M T 1$, and in some of these cases, emission of MeSA, and occasionally of MeBA, was also detected. The highest rates of MeSA emission that we have observed occurred when detached leaves were treated with alamethicin, a channel-forming peptide that has been previously used to mimic the effect of pathogen attack on gene expression and cellular functions, as pathogenic fungi are known to secret pore-forming agents (Brewer et al., 1987). Herbivory by $P$. xylostella larvae for $30 \mathrm{~h}$, which caused extensive damage to the leaves (approximately $40-70 \%$ of the leaf material was consumed by the larvae), resulted in lower rates of MeSA emission per gram FW, as well as some low levels of MeBA emission. Both alamethicin application and $P$. xylostella caused clear induction of AtBSMT1 transcripts.

Physical damage to leaves, and the complex treatment that involved the uprooting of the plant followed by placing the roots in water (a treatment that caused major damage to the roots as well as exposing the roots to a change in osmotic pressure and inorganic ion concentration in the medium and to light), resulted in quick but transient induction of AtBSMT1 (Figure 3b). Additional types of treatments, including flooding the roots of intact plants with distilled water or a saline solution, resulted in only a relatively weak change in AtBSMT1 gene expression (Chen and Pichersky, unpublished). All these abiotic treatments led to only low to undetectable levels of MeSA emissions, a result that is similar to the results of Van Poecke et al. (2001), who failed to detect emission of benzenoid esters from mechanically damaged plants. Strong local induction was observed around areas damaged by piercing-sucking thrips (Figure 4d), but again emission of benzenoid esters, if it occurred, was below detection limits, most likely because of the small area involved. SA, a known global inducer of some defense responses that is also a substrate of AtBSMT1, did not induce AtBSMT1 gene expression, while MeJA, another inducer of systemic defense responses (Seo et al., 2001), did. In both these cases, however, no MeSA emission could be detected.

Van Poecke et al. (2001) suggested that MeSA emission in A. thaliana plays a role in attracting predators of herbivorous insect pests. Our observations of short-lived 
AtBSMT1 induction in response to abiotic stress, such as wounding, and the lack of detectable emission of MeSA and MeBA but the strong localized induction of AtBSMT1 expression around the site of feeding by piercing-sucking thrips suggest additional roles for these highly toxic compounds, perhaps in direct defense.

\section{Experimental procedures}

\section{Plant materials and treatments}

Arabidopsis thaliana (L.) Heynh (ecotype Columbia) plants were grown on soil at $23^{\circ} \mathrm{C}$ under $16 \mathrm{~h}$ light $/ 8 \mathrm{~h}$ dark up to $4-6$ weeks. For selection of transgenic plants, seeds were sterilized in $75 \%$ ethanol for $15 \mathrm{~min}$, rinsed with sterilized water, and germinated on MS medium containing $50 \mu \mathrm{g} \mathrm{ml}^{-1}$ hygromycin. Hygromycin-resistant seedlings were transferred to soil and later confirmed by PCR. The A. lyrata ssp. lyrata plants used in this report were descended from accessions collected in North Carolina (seeds kindly provided by Dr Charles Langley, the University of California, Davis, USA). Plants were grown on soil at $23^{\circ} \mathrm{C}$ under $20 \mathrm{~h}$ light $/ 4 \mathrm{~h}$ dark to induce flowering.

All treatments were carried out with 4- to 6-week-old non-bolting plants of $A$. thaliana. For feeding experiments with $P$. xylostella (kindly provided by T. Mitchell-Olds), 6-week-old rosette plants with their root balls wrapped in aluminum foil were placed in 3-I bell jars. Approximately 50 three-fourths instar larvae were applied per plant and allowed to feed for $30 \mathrm{~h}$, leading to an estimated consumption of total leaf area of $40-70 \%$. For alamethicin (Sigma, St Louis, MO, USA) treatment, leaves were cut off from the base of the petiole and stood up in a small glass beaker containing $10 \mathrm{ml}$ of $5 \mu \mathrm{g} \mathrm{ml}^{-1}$ alamethicin (dissolved 1000 -fold in tap water from a $5 \mathrm{mg} \mathrm{ml}^{-1}$ stock solution in $100 \%$ methanol). Only the petiole of each leaf was submerged in the solution. The glass beaker was then sealed with Saran wrap and placed in a growth chamber. As a control treatment, leaves were placed in tap water only (including $0.1 \%$ methanol). For wounding treatment, two lateral incisions to each side of the midvein of leaves were made with sterile razor blade. Uprooting treatment consisted of removing the entire plant from the soil, rinsing off the soil, and placing the plant in a small beaker with the roots submerged in water. For MeJA treatment, two pots with a total of eight plants were placed in a 5-I glass jar. MeJA ( $1 \mu \mathrm{l}$ ) was dissolved in $200 \mu \mathrm{l}$ ethanol and applied on cotton tips placed in the jar, and the jar was sealed. For SA treatment, SA was dissolved in $0.1 \%$ Tween $(\mathrm{pH} 7.0)$ at a final concentration of $5 \mathrm{mM}$ and used to spray the plants. For treatment with thrips, the plants were placed in a thrip-infested growth chamber for 2 weeks.

\section{Detection of volatile emission from detached leaves treated with alamethicin}

Emitted volatiles were collected by a closed-loop stripping method (Chen et al., 2003; Donath and Boland, 1995) in a controlled climate chamber $\left(23^{\circ} \mathrm{C}, 70 \%\right.$ humidity, $150 \mu \mathrm{mol} \mathrm{m}{ }^{-2} \mathrm{sec}^{-1}$ photosynthetically active radiation (PAR)). After treatment with alamethicin for $21 \mathrm{~h}$ (10 $\mathrm{h}$ light, $11 \mathrm{~h}$ dark) as described in the previous section, the glass beaker with the leaves in alamethicin solution was placed in a 1-I bell jar. For the $P$. xylostella treatment, plants were placed as described above in 3-I bell jars and volatiles were collected during 21-30 $\mathrm{h}$ of larvae feeding. Volatiles were eluted from the trap with $40 \mu \mathrm{CH}_{2} \mathrm{Cl}_{2}$, and $120 \mathrm{ng}$ nonyl acetate was added as an internal standard.
Samples from volatile collections were analyzed on a HewlettPackard 6890 gas chromatograph coupled to a Hewlett-Packard 5973 quadrupole mass selective detector. Separation was performed on (5\%-phenyl)-methylpolysiloxane (DB5) or polyethylene glycol (DB-wax) columns (J\&W Scientific, Folsom, CA, USA) of 30 $\mathrm{m} \times 0.25 \mathrm{~mm}$ i.d. $\times 0.25 \mathrm{~m}$ thickness. Helium was the carrier gas (flow rate $\left.2 \mathrm{ml} \mathrm{min}^{-1}\right)$, a splitless injection $(2 \mu \mathrm{l}$ ) was used, and a temperature gradient of $5^{\circ} \mathrm{C} \mathrm{min}^{-1}$ from $40^{\circ} \mathrm{C}$ (3-min hold) to $240^{\circ} \mathrm{C}$ was applied.

The identities of MeSA and MeBA were determined by comparison of retention time and mass spectra to those of authentic standards and to mass spectra in the NIST and Wiley libraries. For quantification, representative single ion peaks of each compound were integrated and compared to the equivalent response of the internal standard (single ion method).

\section{Determination of gene expression by $R T-P C R$}

The method used is essentially the same as that previously reported by Chen et al. (2003). Primers for $\beta$-tubulin (F: CTCAAGAGGTTCTCAGCAGTA，R: TCACCTTCTTCATCCGCAGTT) and actin-8 (F: ATGAAGATTAAGGTCGTGGCAC, R: GTTTTTATCCGAGTTTGAAGAGGC) were used to judge equality of the concentration of the cDNA templates in each pair of experiments of a treatment-control combination (for $\beta$-tubulin, the amount of cDNA used in the reaction was reduced fivefold, and the number of cycles reduced to 26 , to avoid saturation). The $\beta$-tubulin and actin-8RT-PCR experiments showed less than threefold variation among samples, and based on the $\beta$-tubulin and actin- 8 results, each set of the RTPCR experiments with the AtSABATH primers was normalized accordingly, loaded, and separated on a $1 \%$ agarose gel. Each experiment was repeated at least two times, with similar results.

\section{AtBSMT1 cDNA cloning}

AtBSMT1 was cloned from alamethicin-treated $A$. thaliana leaves by RT-PCR. AtBSMT1 was first cloned based on predictions from GenBank using two primers 5'-AATGGCCAAACCAGTCTTGG-3' and $5^{\prime}$-TCTTACTTCTTAGTCAAGGAAACG-3'. The protein had no activity with any of the substrates tested. As the protein encoded by this CDNA was relatively shorter at the $\mathrm{N}$-terminal end compared to other known SAMT and SAMT-like proteins, 5'-RACE using SMART ${ }^{\text {TM }}$ RACE cDNA Amplification Kit (Clontech Laboratories, Palo Alto, CA, USA) was performed with the At3g11480specific oligonucleotide $5^{\prime}$-GGAGGCTATTGCGAGAGAAGAGCCTGG-3'. The sequence of the $5^{\prime}$-RACE product was compared to the genomic sequence of the gene, and the ATG start was determined. Next, a new forward primer 5'-AATGGATCCAAGATTCATCAACACC- $3^{\prime}$ was made based on the newly identified start of the open-reading frame and used with the original reverse primer for RT-PCR cloning of the full-length AtBSMT1 cDNA.

\section{AIBSMT1 cDNA isolation}

The isolation of AIBSMT1 was based on its homology to AtBSMT1. The same two primers that were used to amplify the full-length AtBSMT1 were also used in an RT-PCR experiment to amplify an AIBSMT1 cDNA from leaf RNA of $A$. lyrata.

\section{RNA gel blot analysis}

RNA gel blot analysis was performed as previously described by Chen et al. (2003) and D'Auria et al. (2002). 
Construction of the AtBSMT1 promoter fusion to the $\beta$-glucuronidase (GUS) reporter gene and histochemical localization of GUS activity

The AtBSMT1 promoter region (1.4 kbp) was isolated via PCR from $A$. thaliana genomic DNA using the primers $5^{\prime}$-CTCCGATTATTGAATTCTAGGCCGGATTG- $3^{\prime}$ and $5^{\prime}$-GAATCTTGGATCCATGGCAATTATCGTAC-3', which contain an EcoRI and an Ncol site, respectively. The resulting PCR product was cloned into pCRT7/ CT-TOPO vector and sequenced. The promoter region of AtBSMT1 was next cut out of the PCRT7/CT-TOPO vector by digestion with $E c o R I$ and $\mathrm{Ncol}$, and the fragment was isolated and inserted into the binary vector pCAMBIA1303 (Hajdukiewicz et al., 1994) by replacing the EcoRI-Ncol fragment containing the CaMV $35 \mathrm{~S}$ promoter. The construct was introduced into Agrobacterium tumefaciens strain GV3101, which was used to transform $A$. thaliana by floral vacuum infiltration (Bechtold et al., 1993). Transgenic lines transformed with the construct were selected using hygromycin, and confirmed by PCR.

The substrate 5-bromo-4-chloro-3-indolyl $\beta$-glucuronide (XGluc) was used to determine the localization of the enzyme activity of the GUS gene (Jefferson et al., 1987). Tissue samples were incubated at $37^{\circ} \mathrm{C}$ in GUS-staining buffer $(50 \mathrm{mM}$ sodium phosphate, $\mathrm{pH} 7.0,0.1 \%$ Triton X-100, and $0.5 \mathrm{mg} \mathrm{ml}^{-1} \mathrm{X}$-Gluc) overnight. After detection of the blue color, chlorophyll was extracted with $70 \%$ ethanol for $24 \mathrm{~h}$.

\section{Biochemical procedures}

Preparation of crude cell-free extracts from E. coli, enzyme assays, protein purification, determination of kinetic properties, and molecular mass estimation were performed as previously described by D'Auria et al. (2002).

\section{Acknowledgements}

We thank Dr Howard Russell at the Diagnostic Services of Michigan State University for the identification of the thrips. This work was supported by a National Science Foundation (NSF) grants No. MCB-9974463 and MCB-0312466 to E.P., by funds from the Max Planck Society to J.G., and by funds from the Salk Institute for Biological Studies and NSF grant No. MCB-0312449 to J.P.N.

\section{References}

Bechtold, N., Ellis, J. and Pelletier, G. (1993) In planta Agrobacterium-mediated gene transfer by infiltration of adult Arabidopsis thaliana plants. Comptes. Rendus. Acad. Sci. Ser. III Sci. Vie-Life. Sci. 316, 1194-1199.

Brewer, D., Mason, F.G. and Taylor, A. (1987) The production of alamethicins by Trichoderma spp. Can. J. Microbiol. 33, 619-625.

Chen, F., Tholl, D., D'Auria, J.C., Farooq, A., Pichersky, E. and Gershenzon, J. (2003) Biosynthesis and emission of terpenoid volatiles from Arabidopsis flowers. Plant Cell, 15, 481-494.

D'Auria, J.C., Chen, F. and Pichersky, E. (2002) Characterization of an acyltransferase capable of synthesizing benzylbenzoate and other volatile esters in flowers and damaged leaves of Clarkia breweri. Plant Physiol. 130, 466-476.

D'Auria, J.C., Chen, F. and Pichersky, E. (2003) The SABATH family of MTs in Arabidopsis thaliana and other plant species. In Recent Advances in Phytochemistry, Vol. 37. (Romeo, J.T., ed.). Oxford: Elsevier Science Ltd., pp. 253-283.
Demirci, F., Demirci, B., Baser, K.H.C. and Guven, K. (2000) The composition and antifungal bioassay of the essential oils of different Betula species growing in Turkey. Chem. Nat. Compd. 36, 159-165.

Donath, J. and Boland, W. (1995) Biosynthesis of acyclic homoterpenes: enzyme selectivity and absolute configuration of the nerolidol precursor. Phytochemistry, 39, 785-790.

Dudareva, N., Murfitt, L.M., Mann, C.J., Gorenstein, N., Kolosova, N., Kish, C.M., Bonham, C. and Wood, K. (2000) Developmental regulation of methyl benzoate biosynthesis and emission in snapdragon flowers. Plant Cell, 12, 949-961.

Engelbert, J., Koch, T., Schuler, G., Bachmann, N., Rechtenbach, J. and Boland, W. (2001) lon channel-forming alamethicin is a potent elicitor of volatile biosynthesis and tendril coiling. Cross talk between jasmonate and salicylate signaling in lima bean. Plant Physiol. 125, 369-377.

Frick, S. and Kutchan, T.M. (1999) Molecular cloning and functional expression of $O$-methyltransferases common to isoqhinoline alkaloid and phenylpropanoid biosynthesis. Plant J. 17, 329-339.

Fukami, H., Asakura, T., Hirano, H., Abe, K., Shimomura, K. and Yamakawa, T. (2002) Salicylic acid carboxyl methyltransferase induced in hairy root cultures of Atropa belladonna after treatment with exogeneously added salicylic acid. Plant Cell Physiol. 43, 1054-1058.

Gang, D.R., Lavid, N., Zubieta, C., Chen, F., Beuerle, T., Lewinsohn, E., Noel, J.P. and Pichersky, E. (2002) Characterization of phenylpropene $O$-methyltransferases from sweet basil: facile change of substrate specificity and convergent evolution within a plant OMT family. Plant Cell, 14, 505-519.

Hajdukiewicz, P., Svab, Z. and Maliga, P. (1994) The small, versatile, pZIP family of Agrobacterium binary vectors for plant transformation. Plant Mol. Biol. 25, 989-994.

Hunter, W.B. and Ullman, D.E. (1992) Anatomy and ultrastructure of the piercing-sucking mouthparts and paraglossal sensilla of Frankliniella occidentalis (Pergande) (Thysanoptera, Thripidae). Int. J. Insect Morph. Embryol. 21, 17-35.

Jefferson, R.A., Kavanagh, T.A. and Bevan, M.W. (1987) Gus fusions - $\beta$-glucuronidase as a sensitive and versatile gene fusion marker in higher plants. EMBO J. 6, 3901-3907.

Knudsen, J.T., Tollsten, L. and Bergstrom, L.G. (1993) Floral scents - a checklist of volatile compounds isolated by headspace techniques. Phytochemistry, 33, 253-280.

Koch, M.A., Haubold, B. and Mitchell-Olds, T. (2000) Comparative evolutionary analysis of chalcone synthase and alcohol dehydrogenase loci in Arabidopsis, Arabis, and related genera (Brassicaceae). Mol. Biol. Evol. 17, 1483-1498.

Kolosova, N., Gorenstein, N., Kish, C.M. and Dudareva, N. (2001) Regulation of circadian methyl benzoate emission in diurnally and nocturnally emitting plants. Plant Cell, 13, 2333-2347.

Lindberg, C.M., Melathopoulos, A.P. and Winston, M.L. (2000) Laboratory evaluation of miticides to control Varroa jacobsoni (Acari: Varroidae), a honey bee (Hymenoptera: Apidae) parasite. J. Econ. Entomol. 93, 189-198.

Murfitt, L.M., Kolosova, N., Mann, C.J. and Dudareva, N. (2000) Purification and characterization of $S$-adenosyl-L-methionine: benzoic acid carboxyl methyltransferase, the enzyme responsible for biosynthesis of the volatile ester methyl benzoate in flowers of Antirrhinum majus. Arch. Biochem. Biophys. 382, $145-151$.

Negre, F., Kolosova, N., Knoll, J., Kish, C.M. and Dudareva, N. (2002) Novel S-adenosyl-L-methionine: salicylic acid carboxyl methyltransferase, an enzyme responsible for biosynthesis of methyl salicylate and methyl benzoate, is not involved in floral 
scent production in snapdragon flowers. Arch. Biochem. Biophys. 406, 261-270.

Noel, J.P., Dixon, R.A., Pichersky, E., Zubieta, C. and Ferrer, J.L. (2003) Structural, functional, and evolutionary basis for methylation of plant small molecules. In Recent Advances in Phytochemistry, Vol. 37 (Romeo, J.T., ed.). Oxford: Elsevier Science Ltd., pp. 37-58.

Pott, M.B., Pichersky, E. and Piechulla, B. (2002) Evening specific oscillations of scent emission, SAMT enzyme activity, and SAMT mRNA in flowers of Stephanotis floribunda. J. Plant Physiol. 159, 925-934.

Raguso, R.A., Light, D.M. and Pichersky, E. (1996) Electroantennogram responses of Hyles lineata (Sphingidae: Lepidoptera) to volatile compounds from Clarkia breweri (Onagraceae) and other moth-pollinated flowers. J. Chem. Ecol. 22, 1735-1766.

Ross, J.R. (2002) S-Adenosyl-L-Methionine: Salicylic Acid Carboxyl Methyltransferase (SAMT), an Enzyme Involved in Floral Scent and Plant Defense in Clarkia Breweri. PhD Thesis. Ann Arbor: University of Michigan.

Ross, J.R., Nam, K.H., D'Auria, J.C. and Pichersky, E. (1999) Sadenosyl-L-methionine: salicylic acid carboxyl methyltransferase, an enzyme involved in floral scent production and plant defense, represents a new class of plant methyltransferases. Arch. Biochem. Biophys. 367, 9-16.
Seo, H.S., Song, J.T., Cheong, J.J., Lee, Y.H., Lee, Y.W., Hwang, I., Lee, J.S. and Choi, Y.D. (2001) Jasmonic acid carboxyl methyltransferase: a key enzyme for jasmonate-regulated plant responses. Proc. Natl. Acad. Sci. USA, 98, 47884793.

Shulaev, V., Silverman, P. and Raskin, I. (1997) Airborne signalling by methyl salicylate in plant pathogen resistance. Nature, $\mathbf{3 8 5}$, 718-721.

Thompson, J.D., Gibson, T.J., Plewniak, F., Jeanmougin, F. and Higgins, D.G. (1997) The CLUSTALX windows interface: flexible strategies for multiple sequence alignment aided by quality analysis tools. Nucl. Acids Res. 24, 4876-4882.

Van Poecke, R.M.P., Posthumus, M.A. and Dicke, M. (2001) Herbivore-induced volatile production by Arabidopsis thaliana leads to attraction of the parasitoid Cotesia rubecula: chemical, behavioral, and gene expression analysis. J. Chem. Ecol. 27, 1911-1928.

Wang, J. and Pichersky, E. (1999) Identification of specific residues involved in substrate discrimination in two plant $O$-methyltransferases. Arch. Biochem. Biophys. 368, 172-180.

Zubieta, C., Koscheski, P., Ross, J.R., Yang, Y., Pichersky, E. and Noel, J.P. (2003) Structural basis for substrate recognition in the salicylic acid carboxyl methyltransferase family. Plant Cell, 15, 1704-1716.

Accession numbers: AY224595 for AtBSMT1 and AY224596 for AIBSMT1. 